\title{
Research and Analysis of Quasi-Zero-Stiffness Isolator with Geometric Nonlinear Damping
}

\author{
Qingguo Meng, Xuefeng Yang, Wei Li, En Lu, and Lianchao Sheng \\ School of Mechatronic Engineering, China University of Mining and Technology, Xuzhou, Jiangsu 221116, China \\ Correspondence should be addressed to Xuefeng Yang; hopeasy@163.com
}

Received 17 May 2017; Revised 1 September 2017; Accepted 26 September 2017; Published 18 October 2017

Academic Editor: Enrico Zappino

Copyright (C) 2017 Qingguo Meng et al. This is an open access article distributed under the Creative Commons Attribution License, which permits unrestricted use, distribution, and reproduction in any medium, provided the original work is properly cited.

\begin{abstract}
This paper presents a novel quasi-zero-stiffness (QZS) isolator designed by combining a tension spring with a vertical linear spring. In order to improve the performance of low-frequency vibration isolation, geometric nonlinear damping is proposed and applied to a quasi-zero-stiffness (QZS) vibration isolator. Through the study of static characteristics first, the relationship between force displacement and stiffness displacement of the vibration isolation mechanism is established; it is concluded that the parameters of the mechanism have the characteristics of quasi-zero stiffness at the equilibrium position. The solutions of the QZS system are obtained based on the harmonic balance method (HBM). Then, the force transmissibility of the QZS vibration isolator is analyzed. And the results indicate that increasing the nonlinear damping can effectively suppress the transmissibility compared with the nonlinear damping system. Finally, this system is innovative for low-frequency vibration isolation of rehabilitation robots and other applications.
\end{abstract}

\section{Introduction}

With the development of science and technology, intelligent lightweight small-sized robots can be achieved; however, vibration isolation is still an important problem in robot control, especially the low-frequency vibration isolation. At present, the low-frequency vibration isolation of robots mainly uses bionics, an active and passive combination of methods, which have high cost and complexity [1-4]. By the mechanical vibration theory, the frequency of the traditional passive vibration isolation system is $\sqrt{2}$ times its own natural frequency [5-7]. To achieve a low frequency of $0.5 \sim 70 \mathrm{~Hz}$, with wide frequency domain vibration isolation, the natural frequency of the vibration isolation system can be reduced. According to the natural frequency of the formula, reducing the natural frequency will reduce the system stiffness or increase the load quality, but in fact the system stiffness and load capacity have their limits [8]. With the deep research of vibration isolation systems, a kind of nonlinear quasi-zerostiffness vibration isolation is realized by a linear positivestiffness spring parallel negative-stiffness mechanism, which has excellent characteristics of high static stiffness and low dynamic stiffness and good low-frequency vibration isolation performance [9]. Alabuzhev et al. [10] made a comprehensive exposition of the theory of zero-stiffness vibration isolation and proposed the corresponding design method. Fulcher et al. [11, 12] used two rods that are articulated under axial force as the negative-stiffness mechanism. Liu et al. [13-16] and Zhang et al. $[17,18]$ used similar Euler bars under axial loads as negative-stiffness mechanisms for vibration isolation of precision instruments; it is obvious that negative-stiffness mechanisms have great prospects in precision engineering. Carrella et al. [19-21] studied the static properties of symmetric oblique springs with parallel positive-stiffness springs and their force and displacement transfer rates. Le and Ahn [22] studied the quasi-zero-stiffness characteristics of the system with the horizontal spring parallel vertical spring and applied it to vehicle seat vibration isolation. On this basis, it is possible to adjust the vibration isolation system with different bearings [23]. The quasi-zero stiffness was realized by using a parallel spring and vertical spring in parallel by Peng et al. [24-26]. Lu and Bai [5] adopted a new kind of connecting rod spring mechanism as the negative-stiffness mechanism, which increased the static bearing capacity of the system. Meng [27] used the disc spring as the negativestiffness mechanism and realized the quasi-zero-stiffness 


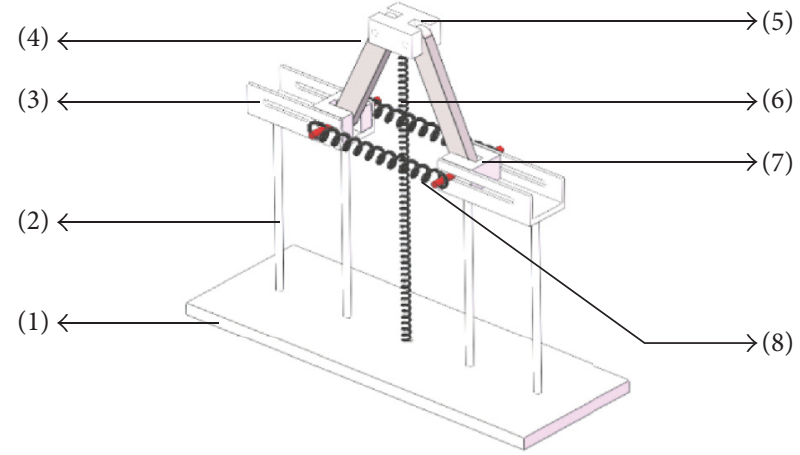

(a) Three-dimensional vibration isolation diagram: (1) base, (2) support column, (3) a skateboard, (4) a connecting rod, (5) stage, (6) vertical springs, (7) slider, and (8) tension spring

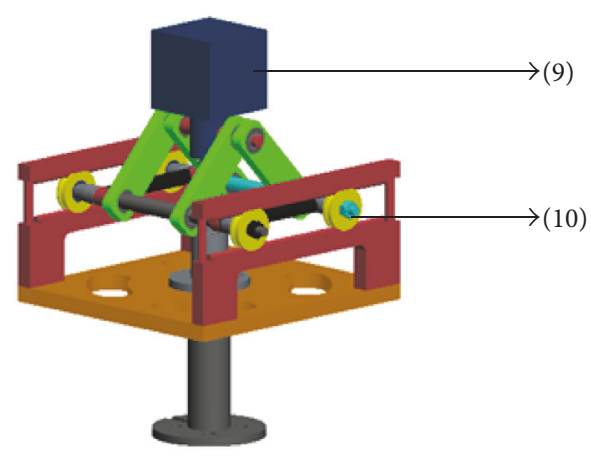

(b) 3D modeling of the vibration isolator: (9) isolated objects and (10) rollers

FiguRE 1

parallel positive-stiffness spring. Zhou et al. introduced a cam-roller negative-stiffness mechanism and also achieved quasi-zero-stiffness isolation [28]. However, there are not many studies on the combination of nonlinear damping and quasi-zero-stiffness systems [29, 30].

In this paper, a QZS vibration isolator with geometric nonlinear damping is developed with the purpose of enhancing the vibration isolation performance. The rest of the paper is organized as follows. The model and static characteristics of the QZS system are built in Section 2. Then, in Section 3, the force transmissibility of the QZS system is analyzed in order to prove the advantage of nonlinear damping in transmissibility. Some conclusions are drawn in Section 4.

\section{3D Modeling of the Vibration Isolator}

As shown in Figure 1(a), we present a vibration isolation system with quasi-zero stiffness, including (1) base, (2) support column, (3) a skateboard, (4) a connecting rod, (5) stage, (6) vertical springs, (7) slider, and (8) tension spring. Two pins are arranged on the slide block, and the slide block, the connecting rod, and the horizontal spring are connected through a cylindrical pin; both ends of the vertical spring are, respectively, arranged in the spring mounting hole of the base and the carrying table. In order to describe the vibration isolator and its vibration isolation performance more clearly, we add a $3 \mathrm{D}$ model of the vibration isolator which is represented in Figure 1(b). In Figure 1(b), (9) represents the mass of the object being isolated, (10) indicates the wheel; it replaces the slider (7) in Figure 1(a), because the use of rollers can reduce the friction, which can improve the accuracy of the model.

For this quasi-zero-stiffness vibration isolation mechanism, the vertical spring is mainly used to carry the static mass, while the two symmetrical horizontal tension springs in the figure are the negative-stiffness mechanism, which can be offset by the rigidity of the vertical positive-stiffness spring.

2.1. Negative-Stiffness Mechanism. If the vertical spring is not considered, the force analysis of the negative-stiffness

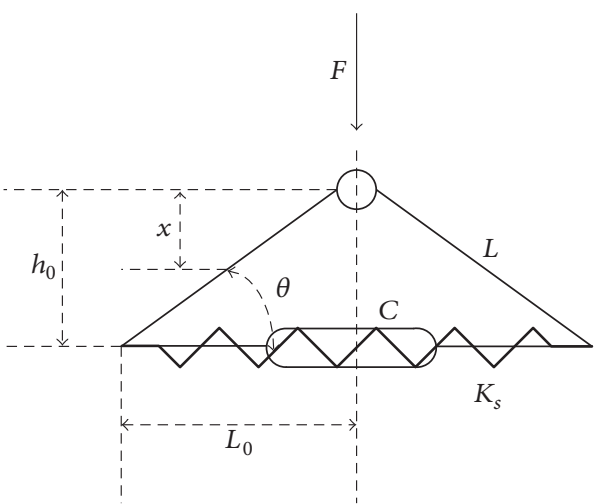

FIGURE 2: Schematic diagram of stress analysis.

mechanism is shown in Figure 2. When the carrier carries a mass of the object, a force $F$ is applied in the vertical direction, and the displacement $X$ of the object causes the two horizontal tension springs to be stretched, and a vertical upward force is generated on the object. According to the geometric relationship, take one of the $1 / 2$ parts of the tensile spring stress analysis and find out the expression of $F$ in the vertical direction:

$$
F=4 F_{s} \tan (\theta),
$$

where $F_{s}=K_{s} \lambda$ is the horizontal force of the $1 / 2$ part of the tension spring, $\theta$ is the angle between the connecting rod and the horizontal line, and $\lambda$ is the distance of the stretched spring $1 / 2$ portion.

In any position, the expression of the angle is

$$
\begin{aligned}
\tan (\theta) & =\frac{h_{0}-x}{\sqrt{L^{2}-\left(h_{0}-x\right)^{2}}}, \\
\lambda & =\sqrt{L^{2}-\left(h_{0}-x\right)^{2}}-L_{0}, \\
h_{0} & =\sqrt{L^{2}-L_{0}^{2}} .
\end{aligned}
$$




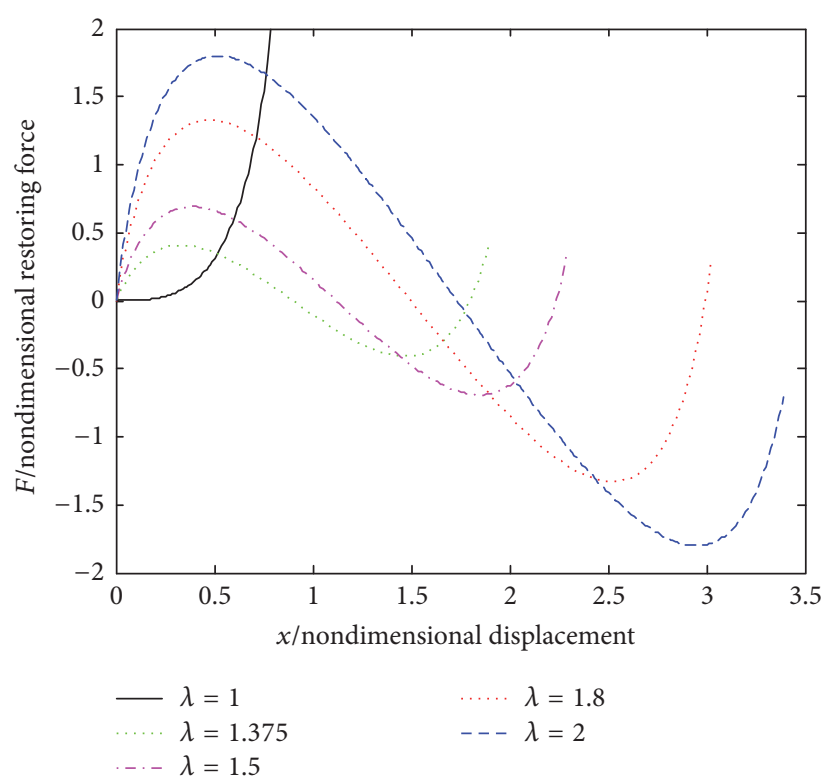

(a) Dimensionless force displacement ( $\lambda$ for different values)

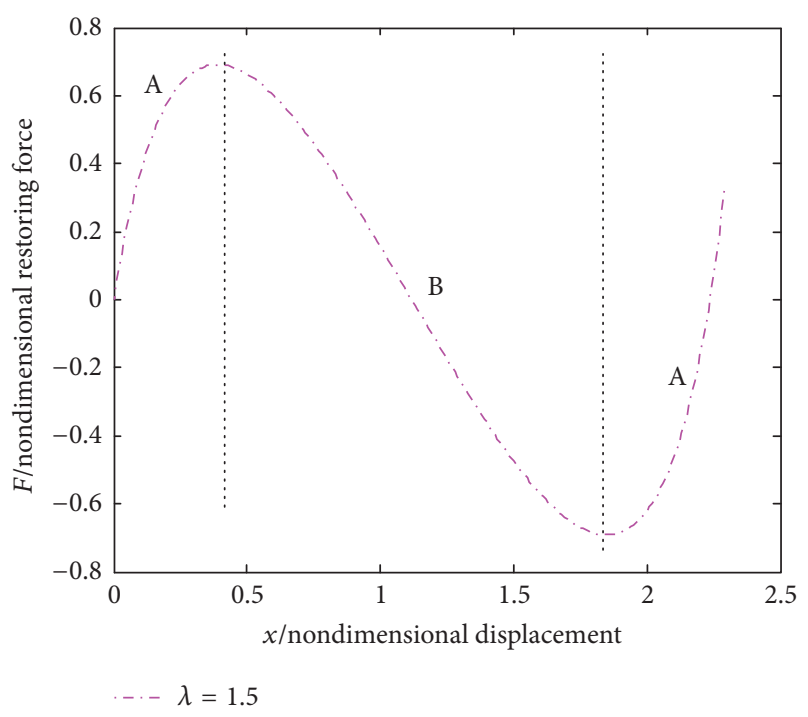

(b) Dimensionless force displacement $(\lambda=1.5)$

FIgURE 3: Dimensionless force displacement.

Combining formulas (1) and (2), the restoring force of the negative stiffness is

$$
\begin{aligned}
F= & 4 K_{s}\left(1-\frac{L_{0}}{\sqrt{L^{2}-\left(\sqrt{L^{2}-L_{0}^{2}}-x\right)^{2}}}\right) \\
& \cdot\left(\sqrt{L^{2}-L_{0}^{2}}-x\right) .
\end{aligned}
$$

Formula (3) is nondimensional and the two sides of the equation are divided by $K_{s} L_{0}$ :

$$
\widehat{F}=4\left(1-\frac{1}{\sqrt{\lambda^{2}-\left(\widehat{h}_{0}-\widehat{x}\right)^{2}}}\right)\left(\widehat{h}_{0}-\widehat{x}\right),
$$

where $\widehat{F}=F / K_{s} L_{0}, \widehat{x}=x / L_{0}, \lambda=L / L_{0}, \widehat{h}_{0}=\sqrt{\lambda^{2}-1}$.

Formula (4) is the expression of the dimensionless force displacement of the negative-stiffness mechanism of the vibration isolation system, so that it can be obtained as shown in Figure 3(a).

According to Figure 3(a), the different values of the parameter $m$ have some influence on the negative-stiffness characteristics of the mechanism. When $\lambda=1$, the dimensionless force always increases with increasing nondimensional displacement, in which case it is not a negative-stiffness mechanism. When $\lambda>1$, this mechanism has negative stiffness, and the negative-stiffness region is between the two extreme points of the displacement curve. As shown in Figure 3(b), the B region is a negative-stiffness region, and the dimensionless force decreases with the increase of the dimensionless displacement in the negative-stiffness region.
2.2. Quasi-Zero-Stiffness (QZS) Vibration Isolation System. In view of the influence of the low-frequency vibration from the ground on the working performance of the biped robot, a new type of quasi-zero-stiffness vibration isolation system is constructed by connecting the negative-stiffness mechanism proposed in Section 2.1 and the linear positivestiffness spring with stiffness $k_{v}$. In order to reduce the vibration of the robot foot, the design block diagram is shown as in Figure 4.

In Figure 4, the quasi-zero-stiffness mechanism is mounted on the robot's foot, but not directly connected to the ground. To reduce friction, the robot moves through the roller of the foot. Therefore, the quasi-zero-stiffness system is mounted on both sides of the wheel to sense low-frequency vibrations from the ground. The robot's foot is extracted and the quasi-zero-stiffness isolator is analyzed. Assuming the vertical force $F$, according to Figure 1, the nondimensional force-displacement relationship of the quasi-zero-stiffness vibration isolation system is expressed as

$$
\widehat{F}=\widehat{x}+4 \alpha\left(1-\frac{1}{\sqrt{\lambda^{2}-\left(\widehat{h}_{0}-\widehat{x}\right)^{2}}}\right)\left(\widehat{h}_{0}-\widehat{x}\right) .
$$

From the above formula, when $\widehat{h}_{0}=\widehat{x}$, the system's return force is not zero, and the system force-displacement relationship is a cubic polynomial, and the displacement belongs to $\widehat{x} \in\left(0 \sim 2 \widehat{h}_{0}\right)$.

Hence, $\widehat{u}=\widehat{x}-\widehat{h}_{0}$. The dimensionless force-displacement expression of the system is

$$
\widehat{F}=\widehat{u}+\widehat{h}_{0}-4 \alpha\left(1-\frac{1}{\sqrt{\lambda^{2}-\widehat{u}^{2}}}\right) \widehat{u},
$$



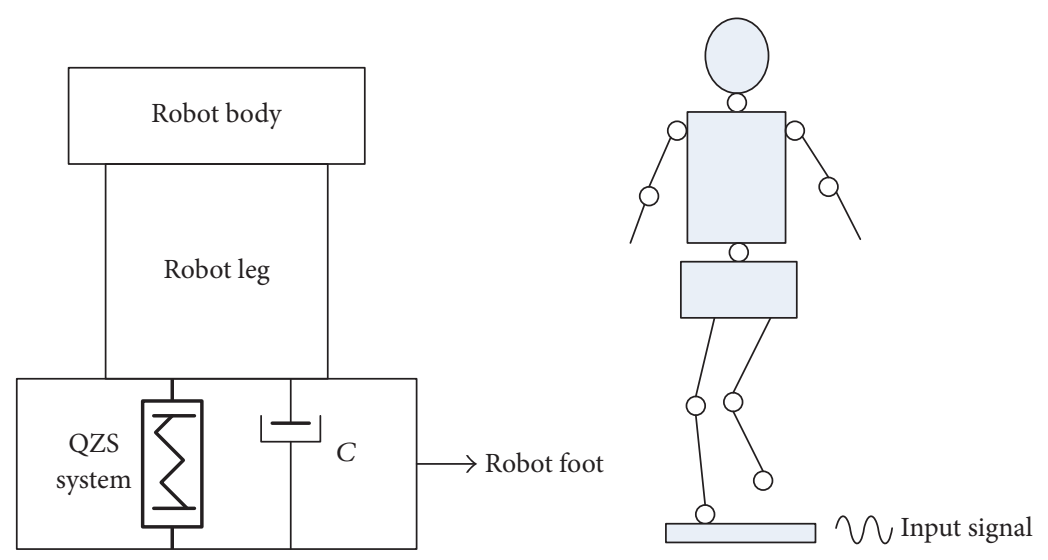

FIGURE 4: Block diagram of rehabilitation robot quasi-zero-stiffness vibration isolation and whole sketch map.

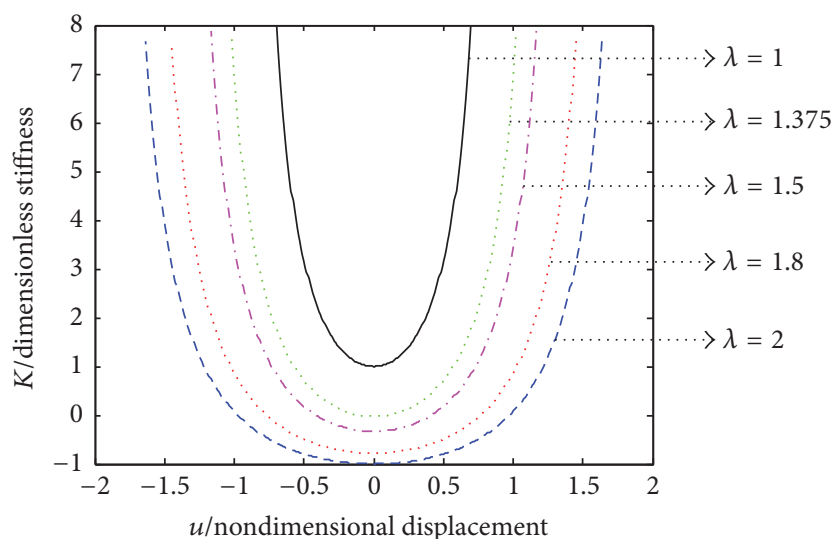

Figure 5: Stiffness curves under different $\lambda$ values.

where $\alpha=K_{s} / K_{v}$ represents the stiffness ratio. Equation (6) is derived. The relationship between the dimensionless stiffness and displacement of the system is obtained:

$$
\widehat{K}=1+4 \alpha\left(\frac{\lambda^{2}}{\left(\lambda^{2}-\widehat{u}^{2}\right)^{3 / 2}}-1\right)
$$

According to formula (7), when $\alpha$ is certain, the effects of different $\lambda$ values on the stiffness are shown in Figure 5. When $\lambda$ is certain, the effect of different stiffness ratios $\alpha$ on the stiffness is shown in Figure 6. Figure 5 shows that when the horizontal tension spring reaches the maximum tensile state, there is minimum stiffness and the stiffness of the system is symmetrical about the equilibrium position. With the increasing of $\lambda$, the dimensionless stiffness changes from positive to negative. Figure 6 shows that when $\lambda$ is constant, the dimensionless stiffness of the system changes from positive to negative as the stiffness ratio increases. In a word, Figures 5 and 6 show that controlling $\lambda$ can change the stiffness and achieve zero stiffness.

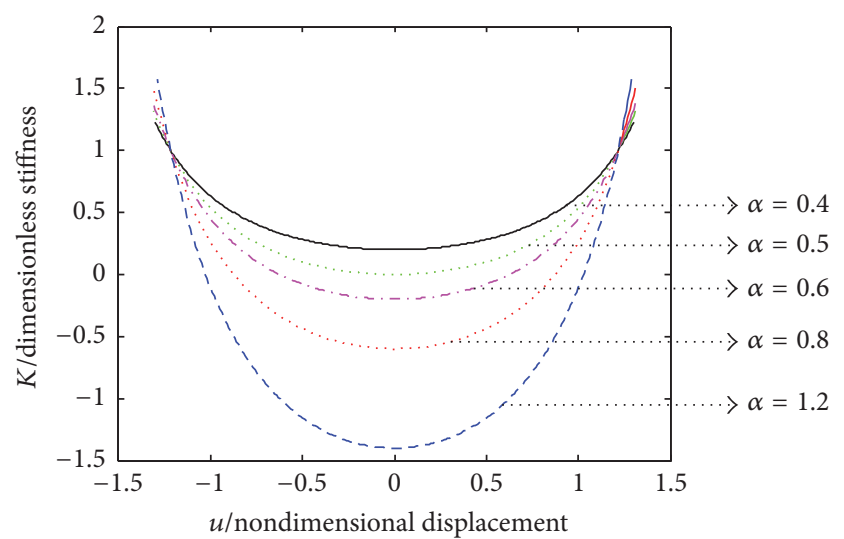

Figure 6: Stiffness curves under different stiffness ratios.

At the equilibrium position, $\widehat{u}=0$, the dimensionless stiffness of the system $\widehat{K}=0$ is obtained:

$$
\begin{aligned}
\widehat{K}_{\mathrm{QZS}} & =1+4 \alpha\left(\frac{1}{\lambda}-1\right), \\
\alpha & =\frac{\lambda}{4(\lambda-1)} .
\end{aligned}
$$

Combined with formulas (7) and (8), the quasi-zero-stiffness characteristics of the system at different positions in the equilibrium position can be obtained, as shown in Figure 7. According to Figure 7, under the situation that $\lambda$ is increasing constantly, we need to have smaller stiffness $\alpha$ to maintain zero stiffness in the equilibrium position.

In order to study the dynamic characteristics of the system, it is necessary to simplify the mathematical model. In this paper, we use the Taylor expansion to approximate the restoring force. Hence, $\widehat{u}=0$. Taylor expansion expression is

$$
\widehat{F}=\widehat{F}(0)+\widehat{F}^{\prime}(0) \widehat{u}+\frac{\widehat{F}^{\prime \prime}(0)}{2 !} \widehat{u}^{2}+\frac{\widehat{F}^{\prime \prime \prime}(0)}{3 !} \widehat{u}^{3}+\cdots
$$




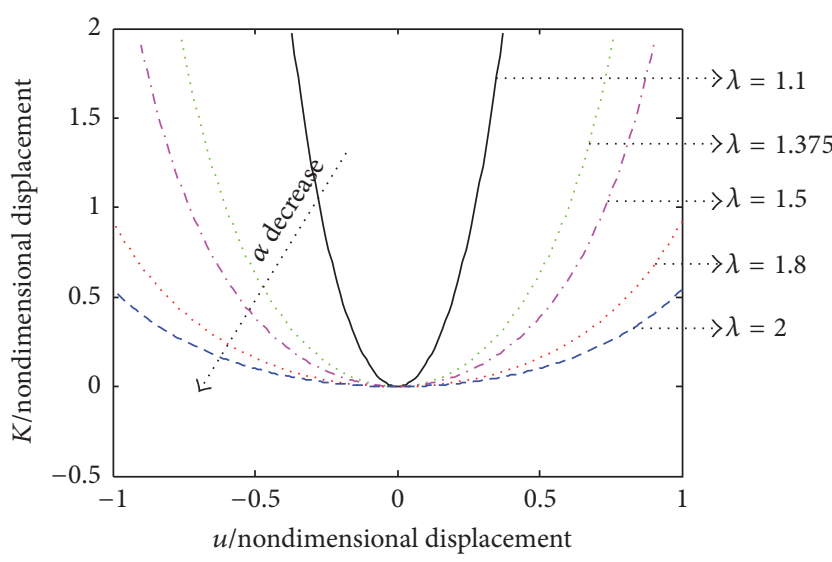

FIGURE 7: Nondimensional displacement stiffness for a parameter.

with the approximate relation of dimensionless force displacement

$$
\widehat{F}=\widehat{F}(0)+\widehat{K}(0) \widehat{u}+\frac{\widehat{K}^{\prime}(0)}{2 !} \widehat{u}^{2}+\frac{\widehat{K}^{\prime \prime}(0)}{3 !} \widehat{u}^{3} .
$$

According to formulas (5) (8) and Figure 5, $\widehat{F}(0)=\widehat{h}_{0}$, $\widehat{K}^{\prime}(0)=0$, and formula (10) is simplified as

$$
\widehat{F}=\widehat{h}_{0}+\left(1+4 \alpha\left(\frac{1}{\lambda}-1\right)\right) \widehat{u}+\frac{2 \alpha}{\lambda^{3}} \widehat{u}^{3} .
$$

When $\lambda=1.375, \alpha=0.92$, and $\chi=2 \alpha / \lambda^{3}$, the exact solution and approximate solution of the dimensionless displacement stiffness are shown in Figure 8. From Figure 8(a), at the equilibrium position $\widehat{u}=0$, the exact curve and the approximate curve of the stiffness curve are coincident, so it is feasible to replace the exact expression in the small amplitude range.

According to Figure 8(a), with the increase of the displacement absolute value, the error of displacement stiffness and the approximate solution is also increasing gradually, so it is necessary to carry out error analysis in the balance position of the system. If the variable $\varepsilon$ (percentage) represents the system's error, the exact expression is

$$
\varepsilon=\left|1-\frac{\left(6 \alpha / \lambda^{3}\right) \widehat{u}^{2}}{1+4 \alpha\left[\left(\lambda^{2} /\left(\lambda^{2}-\widehat{u}^{2}\right)^{3 / 2}-1\right)\right]}\right| * 100 \% .
$$

The stiffness error is related to the geometric parameters, displacement, and stiffness ratio of the system. Therefore, the stiffness of the system is determined as long as the geometric parameters and the stiffness ratio of the system are determined. If $\lambda=1.375$ and $\alpha=0.92$, the curve of stiffness error with displacement is plotted in Matlab, as shown in Figure 8(b).

2.3. Nonlinear Damping Characteristic. The geometric nonlinear damping characteristic is mainly analyzed in this part. Figure 9 shows the transmission path of the damping force from the horizontal direction to the vertical direction.

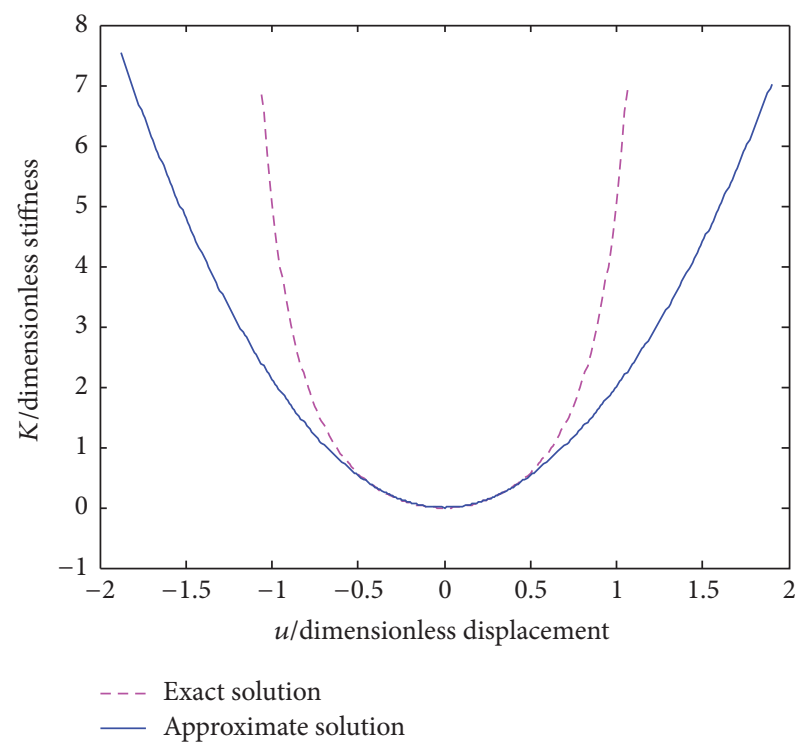

(a) Comparison of exact stiffness and approximate stiffness

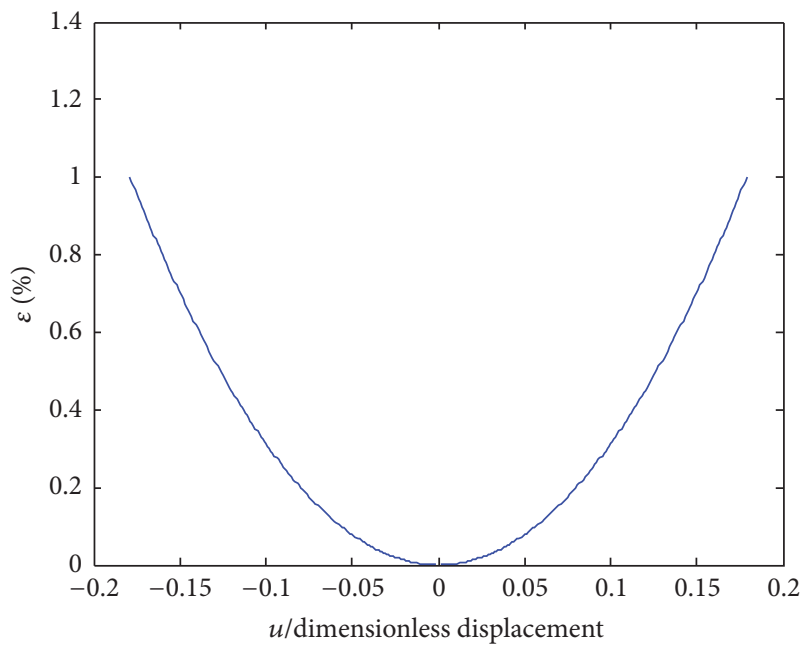

(b) Plane diagram of the error curve of the system's precise and approximate stiffness

FiguRe 8

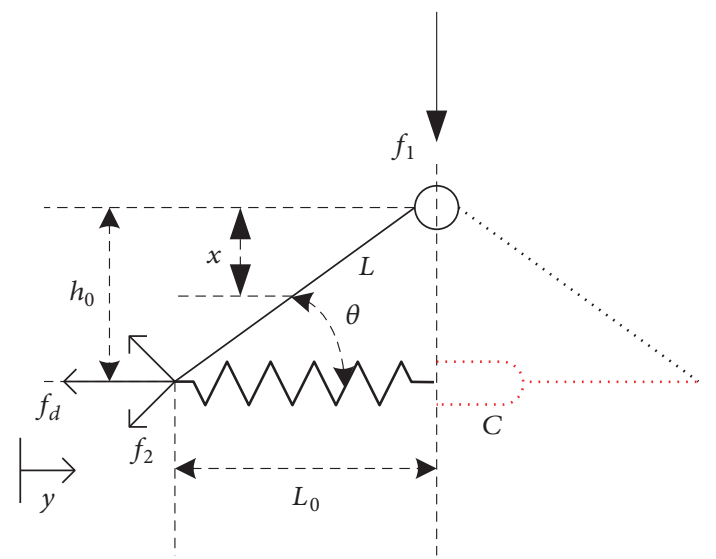

FIGURE 9: Schematic diagram of the damping force transmission path. 
Based on the analysis in the previous subsection, the displacement of the left hinge axis in the horizontal direction is given by

$$
y=L(1-\cos \theta),
$$

where $\cos \theta=\sqrt{4 L^{2}-x^{2}} / 2 L$. By differentiating (12) with respect to time, the velocity of the left hinge axis in the horizontal direction can be obtained:

$$
\dot{y}=\frac{x}{2 \sqrt{4 L^{2}-x^{2}}} \dot{x} .
$$

The right hinge axis velocity is equal to that of the left one, but the direction is opposite. Thus, the horizontal damping force is given by

$$
f_{d}=2 C \dot{y}
$$

and at the same time

$$
\begin{aligned}
& f_{2}=2 f_{1} \sin \theta, \\
& f_{d}=2 f_{1} \cos \theta .
\end{aligned}
$$

Therefore, the nonlinear damping force transmitted to the loading support can be further written as

$$
f_{2}=\frac{C x^{2}}{4 L^{2}-x^{2}} \dot{x}
$$

Writing (16) in a nondimensional form yields

$$
k_{22}=\frac{c \widehat{u}^{2}}{4-\widehat{u}^{2}} \widehat{\dot{u}}
$$

where $k_{22}=f_{2} / K_{v} L$ and $c=C / K_{v}$.

Equation (10) can be approximated by a third-order Taylor series about $u=0$ with the purpose of simplifying the subsequent dynamic analysis:

$$
k_{22 u}=\frac{1}{4} c \widehat{u}^{2} \widehat{\dot{u}}
$$

\section{Force Transmissibility}

3.1. Considering Nonlinear Damping. The equation of motion of the load under harmonic force excitation is given by

$$
m \ddot{x}+c_{0} \dot{x}+f_{2}+f(x)=F \cos (w t) .
$$

Writing (19) in a nondimensional form and replacing the exact restoring force and the nonlinear damping force with their approximations can yield

$$
\widehat{\ddot{u}}+2 \zeta_{1} \widehat{\dot{u}}+\frac{1}{2} \zeta_{2} \widehat{u}^{2} \widehat{\dot{u}}+\chi \widehat{u}^{3}=\gamma \rho \cos (w \tau)
$$

where $w_{0}=\sqrt{K_{v} / m}, \tau=w_{0} t, \zeta_{1}=c_{0} / 2 m w_{0}, \zeta_{2}=c / 2 m w_{0}$, $f=F / K_{v} L$, and $\Omega=w / w_{0}$; when the system is subjected to harmonic force excitation, $\gamma=1, \rho=F$, then (20) can be solved in the following application of the HBM. The solution of (20) is assumed to be of the form

$$
\begin{aligned}
& \widehat{u}=A \cos (w \tau+\varphi), \\
& \widehat{\dot{u}}=-A \Omega \cos (\Omega \tau+\varphi), \\
& \widehat{\ddot{u}}=-A \Omega^{2} \cos (\Omega \tau+\varphi) .
\end{aligned}
$$

Substituting (21), (22), and (23) into (20) yields

$$
\begin{array}{r}
-A \Omega^{2} \cos (\Omega \tau+\varphi)-2 \zeta_{1} A \Omega \sin (\Omega \tau+\varphi) \\
-\frac{1}{2} \zeta_{2} A^{3} \Omega \cos ^{2}(\Omega \tau+\varphi) \sin (\Omega \tau+\varphi) \\
+\chi A^{3} \cos ^{3}(\Omega \tau+\varphi)=\gamma \rho \cos (\Omega \tau) .
\end{array}
$$

The right item of (24) can be deformed into

$$
\begin{aligned}
\cos (\Omega \tau)= & \gamma \rho \cos (\Omega \tau+\varphi-\varphi) \\
= & \gamma \rho \cos (\Omega \tau+\varphi) \cos (\varphi) \\
& +\gamma \rho \sin (\Omega \tau+\varphi) \sin (\varphi) .
\end{aligned}
$$

Combining (24) and (25) can yield

$$
\begin{aligned}
-A \Omega^{2}+\frac{3}{4} \chi A^{3} & =\gamma \rho \cos (\varphi), \\
-2 A \zeta_{1} \Omega-\frac{1}{4} \zeta_{2} A^{3} \Omega & =\gamma \rho \sin (\varphi) .
\end{aligned}
$$

Thus, the amplitude-frequency equation can be derived with the application of $\sin (\varphi)+\cos (\varphi)=1$ :

$$
\left(-A \Omega^{2}+\frac{3}{4} \chi A^{3}\right)^{2}+\left(-2 A \zeta_{1} \Omega-\frac{1}{4} \zeta_{2} A^{3} \Omega\right)^{2}=\gamma^{2} \rho^{2},
$$

where

$$
\begin{aligned}
& \Omega^{f 0}{ }_{1,2} \\
& \quad=\frac{1}{2} \sqrt{\frac{3}{2} \chi A^{2}-4 \xi_{1}{ }^{2}-\zeta_{1} \zeta_{2} A^{2}-\frac{1}{16} \zeta^{2}{ }_{2} A^{4} \pm \sqrt{\Delta}}
\end{aligned}
$$

$\Delta$

$$
\begin{aligned}
= & 16 \zeta_{1}{ }^{4}+8 \zeta_{1}{ }^{3} \zeta_{2} A^{2}+\frac{3}{2} \zeta_{1} \zeta_{2} A^{4}-12 \chi \zeta_{1}{ }^{2} \\
& +\frac{1}{8} \zeta_{1} \zeta_{2}{ }^{2} A^{6}-3 \chi \zeta_{1} \zeta_{2} A^{4}+\frac{1}{256} \zeta_{2}{ }^{4} A^{8} \\
& -\frac{3}{16} \chi \zeta_{2}^{2} A^{6}+\frac{\rho}{A^{2}} .
\end{aligned}
$$

According to the above analysis, the force transmitted to the load is given by

$$
f_{t}=2 \zeta_{1} \widehat{\dot{u}}+\frac{1}{2} \zeta_{2} \widehat{u}^{2} \widehat{\dot{u}}+\chi \widehat{u}^{3}
$$




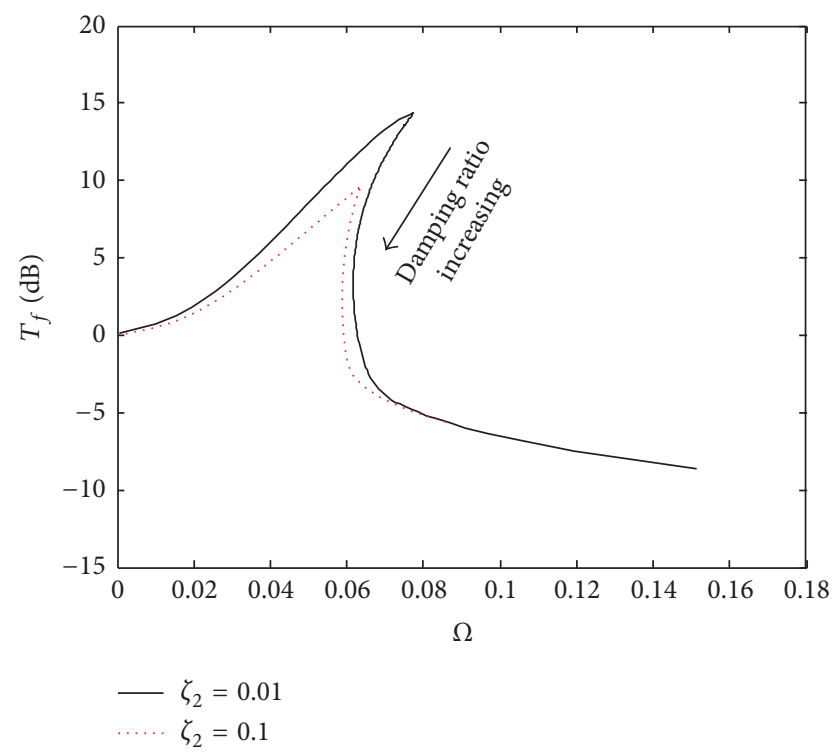

FIgURE 10: Force transmissibility for various $\zeta_{2}$.

Then, the amplitude of the transmitted force can be obtained by substituting (21), (22), and (23) into (30):

$$
F_{t}=\sqrt{\left(\frac{3}{4} \chi A^{3}\right)^{2}+\left(-2 A \zeta_{1} \Omega-\frac{1}{4} \zeta_{2} A^{3} \Omega\right)^{2}} .
$$

Therefore, the force transmissibility of the QZS system can be written as

$$
\begin{aligned}
& T_{f 0}=20 \\
& \cdot \log \left(\frac{\sqrt{\left((3 / 4) \chi A^{3}\right)^{2}+\left(-2 A \zeta_{1} \Omega-(1 / 4) \zeta_{2} A^{3} \Omega\right)^{2}}}{f}\right) .
\end{aligned}
$$

The effect of the damping ratio $\zeta_{2}$ on the force transmissibility is shown in Figure 10. It can be clearly observed that increasing $\zeta_{2}$ can effectively suppress the transmissibility, but it has little influence on the force isolation performance at higher frequencies.

3.2. Without Nonlinear Damping. Without considering the role of nonlinear damping, the dimensionless equations of the system are written as

$$
\widehat{\ddot{u}}+2 \xi_{1} \hat{\dot{u}}+\chi \widehat{u}^{3}=\gamma \rho \cos (\Omega \tau) .
$$

When the system is excited by harmonic force, $\gamma=1$, $\rho=\widehat{F}$, for (33), we can obtain the amplitude-frequency response function of the system by using the harmonic balance method, neglecting the high-order harmonic term and eliminating the phase difference:

$$
\left(\frac{3}{4} \chi A^{3}{ }_{1}-\Omega^{2} A_{1}\right)^{2}+4 \xi^{2} \Omega^{2} A_{1}^{2}=\gamma^{2} \rho^{2}
$$

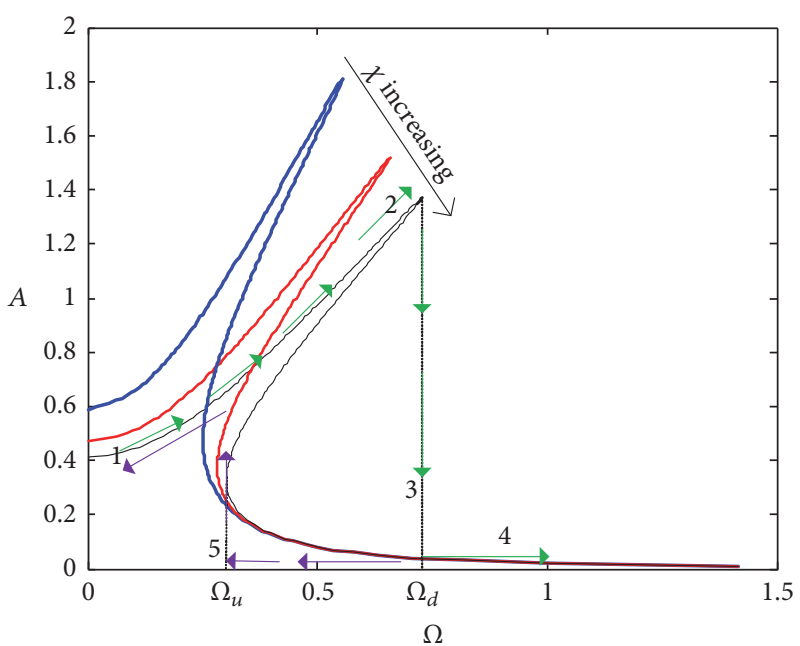

FIGURE 11: The influence of the nonlinear term of quasi-zero stiffness on the $A-\Omega$ curve.

According to (34), two solutions can be solved in harmonic force and harmonic displacement excitation; the expression is

$$
\begin{aligned}
& \Omega^{f}{ }_{1,2} \\
& \quad=\frac{1}{2} \sqrt{3 \chi A^{2}{ }_{1}-8 \xi^{2} \pm \frac{4}{A_{1}} \sqrt{4 \xi^{4} A^{2}{ }_{1}-3 \chi \xi^{2} A^{4}{ }_{1}+\rho^{2}}} .
\end{aligned}
$$

According to the amplitude-frequency characteristic formula (35) of the system, the amplitude of the response is related not only to the frequency ratio, but also to the damping ratio and the nonlinear term. The relation between the nonlinear term and the response amplitude is shown in Figure 11. It can be seen that the vibration amplitude decreases gradually with the increase of the nonlinear term of the system, and the bend of the $A-\Omega$ curve is more and more obvious in the process of nonlinear term increasing; the nonlinear term contained in the quasi-zero-stiffness system can play a role in decreasing the amplitude of the response.

The force transmitted to the foundation by a quasi-zerostiffness vibration isolation system is

$$
\widehat{F}_{t}=\sqrt{f_{e}^{2}+f_{d}^{2}}=\sqrt{\left(\frac{3}{4} \chi A_{1}^{3}\right)^{2}+\left(2 \xi A_{1} \Omega\right)^{2}}
$$

and its force transfer rate is

$$
T_{f}=\frac{\sqrt{\left((3 / 4) \chi A_{1}^{3}\right)^{2}+\left(2 \xi A_{1} \Omega\right)^{2}}}{\widehat{F}} .
$$

According to the above analysis, we can get the influence of different excitation amplitudes and damping parameters on the vibration isolation system, as shown in Figures 12 and 13.

Figure 12 shows a force transmission rate curve, and a green curve indicates a nonstationary solution. From Figure 12, with the increase of the damping ratio, the force 


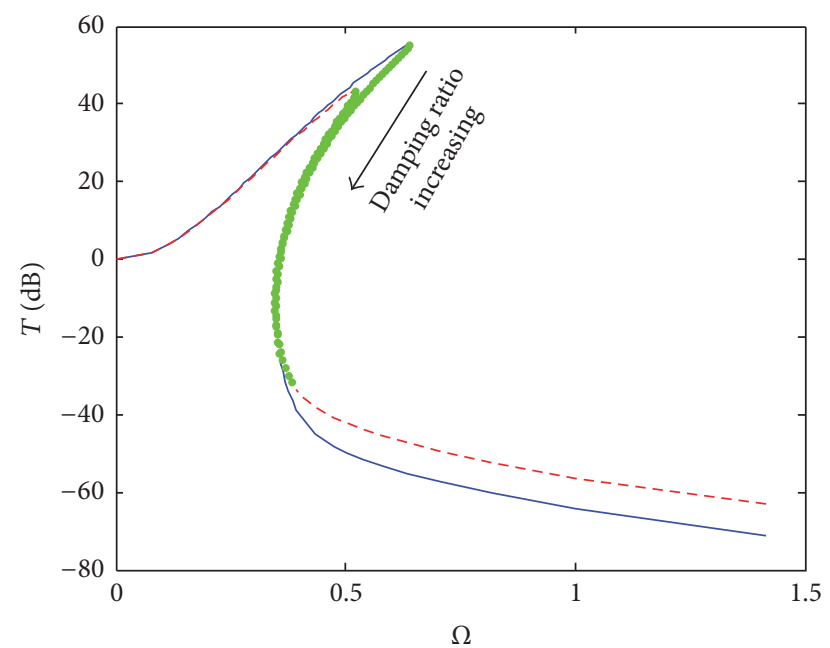

FIGURE 12: Force transfer rate.

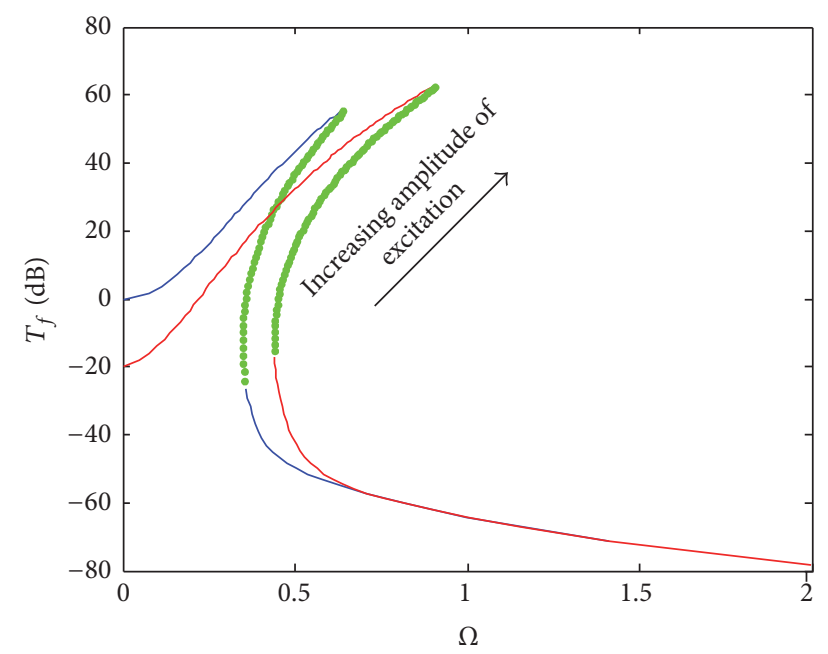

FIGURE 13: Effect of different excitation amplitudes on the quasizero-stiffness force transfer rate.

transfer rate decreases. As shown in Figure 13, the green curve is also an unstable solution. It is concluded that, with the increase of the damping ratio, the transmission rate of the system is also decreasing; that is, the greater the damping, the smaller the transmissibility, which is consistent with the common sense of life. As can be seen from Figure 13, the range of the unstable solution of the system is gradually decreasing in the process of increasing the damping ratio, but in the high-frequency part, the system with relatively large damping has no better vibration isolation than the small system. But from Figures 12 and 13, we can find that, with the existence of geometric nonlinearity in the horizontal direction, the rate of force transfer of the system is obviously decreased.

\section{Conclusions}

(1) A novel type of quasi-zero-stiffness vibration isolation system is designed in this paper. It is formed by the parallel connection of the positive-stiffness spring and the negativestiffness mechanism. The negative-stiffness mechanism consists of two horizontal tension springs and exhibits negativestiffness characteristics during the movement.

(2) Static and dynamic analyses of the novel quasi-zero stiffness are carried out. Through the static analysis of the system, the size of the quasi-zero-stiffness mechanism and the quality of the vibration isolation can be determined. In the dynamic aspect, nonlinear damping is added and analyzed by the theoretical formula, which can reduce the transmissibility of the vibration isolation system.

(3) The quasi-zero-stiffness system with an innovative structure is very important for low-frequency vibration isolation and has important significance. In this paper, the excellent vibration isolation capability of the system is applied to a rehabilitation robot. This is verified theoretically and provides guidance for practical applications.

\section{Conflicts of Interest}

The authors declare that there are no conflicts of interest regarding the publication of this paper.

\section{Acknowledgments}

This work is based upon work supported by the National Natural Science Foundation of China (no. 51305444) and the Project Funded by the Priority Academic Program Development of Jiangsu Higher Education Institutions (PAPD).

\section{References}

[1] A. Hamon and Y. Aoustin, "Study of different structures of the knee joint for a planar bipedal robot," in Proceedings of the 9th IEEE-RAS International Conference on Humanoid Robots, HUMANOIDS09, pp. 113-120, Paris, France, December 2009.

[2] J. Li, H. Zhong, W.-W. Mao, F. Zhou, and Q. Liang, "Active and passive composite vibration damping and impact resistance research for biped robots," Electronic Technique, no. 9, 2015.

[3] W.-W. Mao, F. Zhou, J. Li, and Q. Liang, "Study of active-passive vibration attenuation system for crus of biped robots," Computer Simulation, vol. 33, no. 2, pp. 355-360, 2016.

[4] J. H. Lee, S. Okamoto, H. Koike, and K. Tani, "Development and motion control of a biped walking robot based on passive walking theory," Artificial Life and Robotics, vol. 19, no. 1, pp. 68-75, 2014.

[5] C.-H. Lu and H.-B. Bai, "A new type nonlinear ultra-low frequency passive vibration isolation system," Journal of Vibration and Shock, vol. 30, no. 1, pp. 234-236, 2011.

[6] M. J. Brennan, I. Kovacic, A. Carrella, and T. P. Waters, "On the jump-up and jump-down frequencies of the Duffing oscillator," Journal of Sound and Vibration, vol. 318, no. 4-5, pp. 1250-1261, 2008.

[7] X.-Y. Li and M.-L. Zhang, Mechanical Vibration, Tsinghua University Press, Beijing, China, 2009.

[8] G.-F. Nie and J. Zahng, "Design and characterization of a new quasi zero stiffness vibration isolation system," Journal of Jingchu University of Technology, vol. 31, no. 2, pp. 26-32, 2016.

[9] L.-S. Meng, J.-G. Sun, F. Niu, X.-D. Ren, and J.-C. Qi, “Design and analysis of a novel quasi-zero stiffness vibration isolation 
system," Journal of Vibration and Shock, vol. 33, no. 11, pp. 195199, 2014.

[10] P. Alabuzhev, A. Gritchin, L. Kim et al., Vibration Protecting and Measuring Systems with Quasi-Zero Stiffness, Hemisphere Publishing Corporation, Washington, DC, USA, 1989.

[11] B. A. Fulcher, D. W. Shahan, M. R. Haberman, C. C. Seepersad, and P. S. Wilson, "Analytical and experimental investigation of buckled beams as negative stiffness elements for passive vibration and shock isolation systems," Journal of Vibration and Acoustics, vol. 136, no. 3, Article ID 031009, 12 pages, 2014.

[12] B. A. Fulcher, Evaluation of Systems Containing Negative Stiffness Elements for Vibration and Shock Isolation, The University of Texas at Austin, Austin, Tex, USA, 2012.

[13] X.-T. Liu, J.-Y. Sun, F. Xiao, and H.-X. Hua, "Principle and performance of a quasi-zero stiffness isolator for micro-vibration isolation," Journal of Vibration and Shock, vol. 32, no. 21, pp. 6973, 2013.

[14] X.-T. Liu, Z.-Y. Zhang, and H.-X. Hua, "Characteristics of a novel low-frequency isolator," Journal of Vibration and Shock, vol. 31, no. 5, pp. 161-164, 2012.

[15] X. Huang, X. Liu, J. Sun, Z. Zhang, and H. Hua, "Vibration isolation characteristics of a nonlinear isolator using euler buckled beam as negative stiffness corrector: a theoretical and experimental study," Journal of Sound and Vibration, vol. 333, no. 4, pp. 1132-1148, 2014.

[16] X. T. Liu, X. C. Huang, and H. X. Hua, "On the characteristics of a quasi-zero stiffness isolator using Euler buckled beam as negative stiffness corrector," Journal of Sound and Vibration, vol. 332, no. 14, pp. 3359-3376, 2013.

[17] J.-Z. Zhang, S. Dong, and D. Li, "Study on new type vibration isolation system based on combined positive and negative stiffness," Nanotechnology and Precision Engineering, vol. 2, no. 4, pp. 314-318, 2004.

[18] J. Z. Zhang, D. Li, S. Dong, and M. J. Chen, "Study on ultralow frequency parallel connection isolator used for precision instruments," China Mechanical Engineering, vol. 15, no. 1, pp. 69-70, 2004.

[19] A. Carrella, M. J. Brennan, and T. P. Waters, "Static analysis of a passive vibration isolator with quasi-zero-stiffness characteristic," Journal of Sound and Vibration, vol. 301, no. 3-5, pp. 678689, 2007.

[20] A. Carrella, M. J. Brennan, I. Kovacic, and T. P. Waters, "On the force transmissibility of a vibration isolator with quasi-zerostiffness," Journal of Sound and Vibration, vol. 322, no. 4-5, pp. 707-717, 2009.

[21] A. Carrella, M. J. Brennan, T. P. Waters, and V. Lopes Jr., "Force and displacement transmissibility of a nonlinear isolator with high-static-low-dynamic-stiffness," International Journal of Mechanical Sciences, vol. 55, no. 1, pp. 22-29, 2012.

[22] T. D. Le and K. K. Ahn, "A vibration isolation system in low frequency excitation region using negative stiffness structure for vehicle seat," Journal of Sound and Vibration, vol. 330, no. 26, pp. 6311-6335, 2011.

[23] C.-C. Lan, S.-A. Yang, and Y.-S. Wu, "Design and experiment of a compact quasi-zero-stiffness isolator capable of a wide range of loads," Journal of Sound and Vibration, vol. 333, no. 20, pp. 4843-4858, 2014.

[24] X. Peng and S.-X. Zhang, "Nonlinear resonance response analysis of a kind of passive isolation system with quasi-zero stiffness," Journal of Hunan University Natural Sciences, vol. 38, no. 8, pp. 34-39, 2011.
[25] X. Peng and S.-X. Zhang, "Static and linear dynamic characteristics analysis for a kind of vibration isolation system with quasizero-stiffness," Chinese Quarterly of Mechanics, vol. 33, no. 3, pp. 492-498, 2012

[26] X. Peng, S.-N. Chen, and F.-P. Song, "Research on theory of negative stiffness and its application," Journal of Hunan University, no. 4, pp. 89-94, 1992.

[27] L.-S. Meng, Design and Characteristics Analysis of the Novel Quasi-zero Stiffness Isolator, Academy of Military Medical Sciences, 2015.

[28] J.-X. Zhou, X.-L. Wang, D.-L. Xu, and J. Zhang, "Experimental study on vibration isolation characteristics of the quasi-zero stiffness isolator with cam-roller mechanism," Journal of Vibration Engineering, vol. 28, no. 3, pp. 449-455, 2015.

[29] B. Tang and M. J. Brennan, "A comparison of two nonlinear damping mechanisms in a vibration isolator," Journal of Sound and Vibration, vol. 332, no. 3, pp. 510-520, 2013.

[30] P. F. Guo, Z. Q. Lang, and Z. K. Peng, "Analysis and design of the force and displacement transmissibility of nonlinear viscous damper based vibration isolation systems," Nonlinear Dynamics, vol. 67, no. 4, pp. 2671-2687, 2012. 


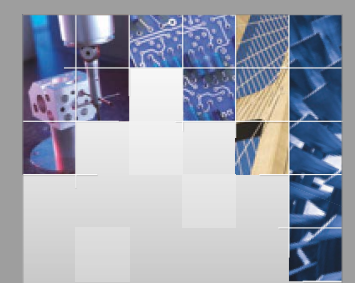

\section{Enfincering}
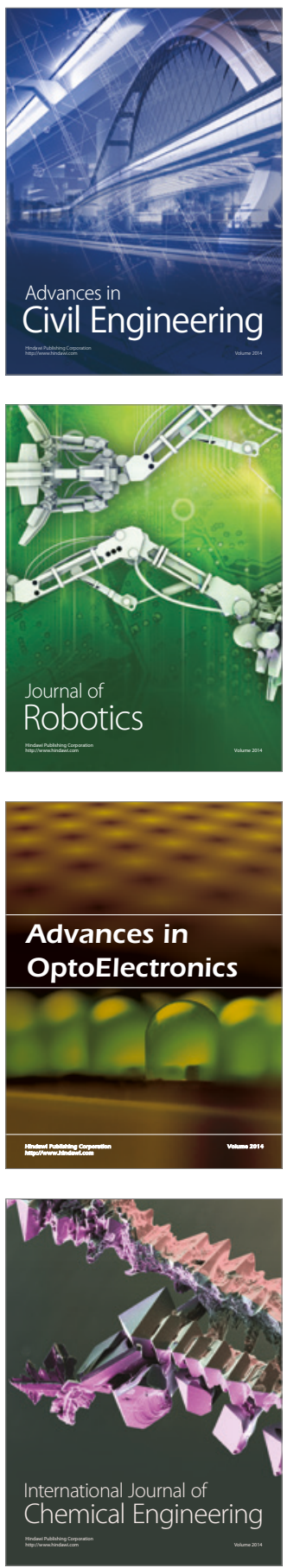

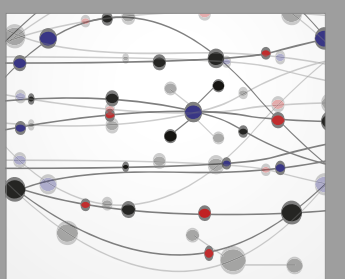

The Scientific World Journal

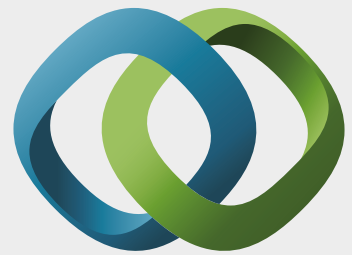

\section{Hindawi}

Submit your manuscripts at

https://www.hindawi.com
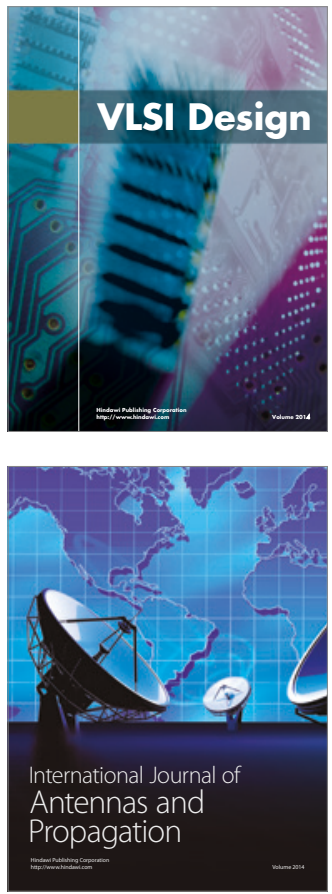

\section{Rotating}

Machinery
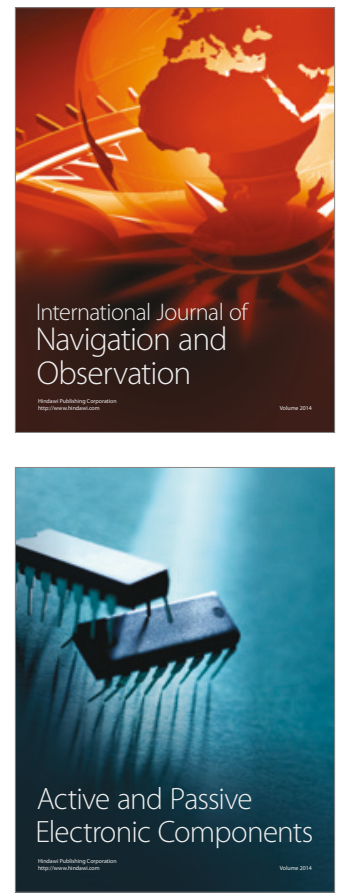
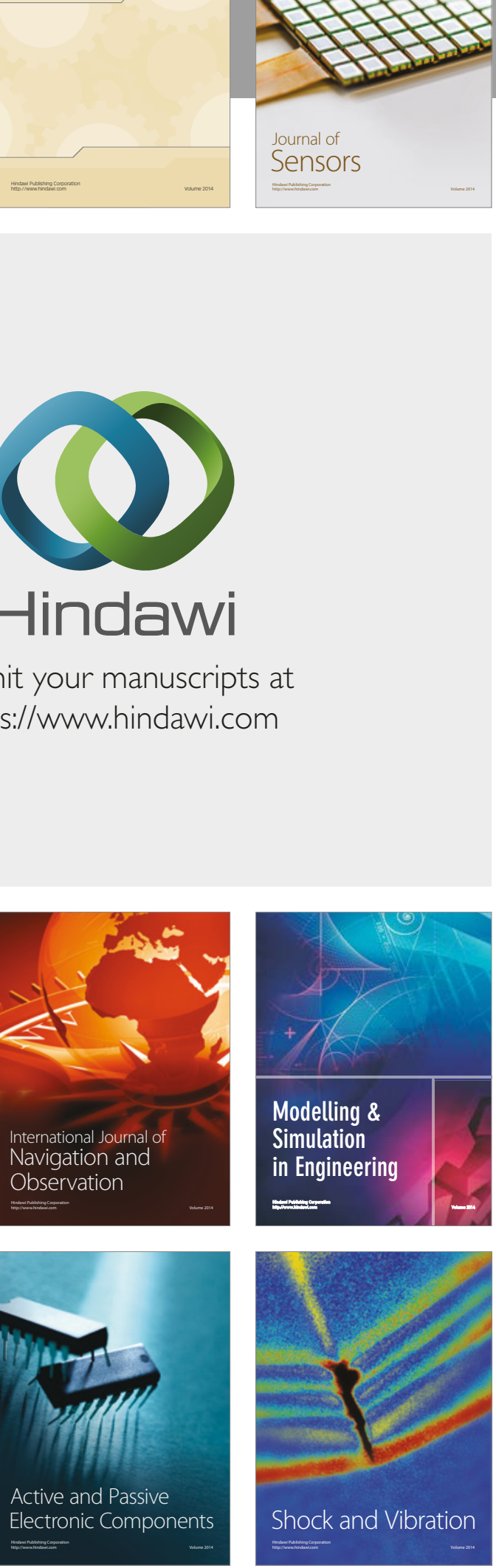
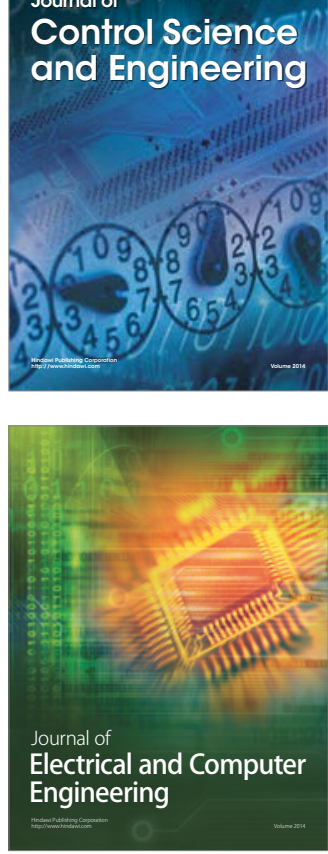

Distributed

Journal of

Control Science

and Engineering
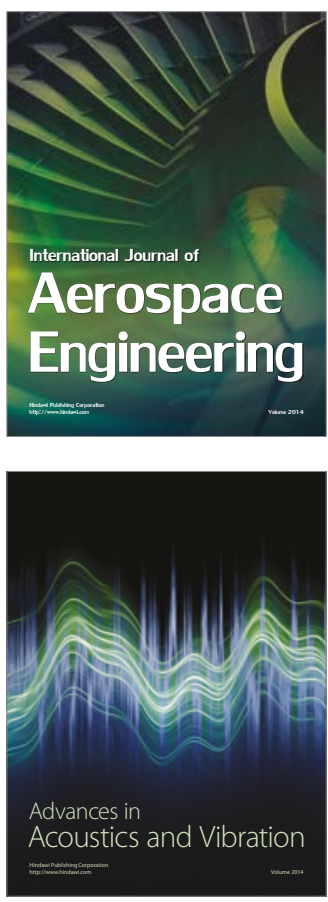

Sensor Networks 